\title{
Game Cross Efficiency for Systems with Two-Stage Structures
}

\author{
Chaoqun Ma, ${ }^{1}$ Debin Liu, ${ }^{1}$ Zhongbao Zhou, ${ }^{1}$ Wei Zhao, ${ }^{1}$ and Wenbin Liu ${ }^{1,2}$ \\ ${ }^{1}$ School of Business Administration, Hunan University, Changsha 410082, China \\ ${ }^{2}$ Kent Business School, University of Kent, Canterbury CT2 7PE, UK \\ Correspondence should be addressed to Debin Liu; liudebinhnu@163.com
}

Received 14 December 2013; Accepted 13 January 2014; Published 4 March 2014

Academic Editor: Pu-yan Nie

Copyright (C) 2014 Chaoqun Ma et al. This is an open access article distributed under the Creative Commons Attribution License, which permits unrestricted use, distribution, and reproduction in any medium, provided the original work is properly cited.

\begin{abstract}
Data envelopment analysis (DEA) is a nonparametric approach for measuring the relative efficiencies of peer decision-making units (DMUs). For systems with two-stage structures, where all the outputs from the first stage are the only inputs to the second stage, the centralized model, which is based on the concept of cooperative game theory, has been widely used to examine the efficiencies of such systems. We define the cross efficiencies of systems with two-stage structures. Since the centralized model may lead to multiple and unacceptable cross efficiencies and rankings of DMUs due to its high flexibility in choosing optimal weights on input and output factors, we develop a game model to obtain a unique cross efficiency measure, which is constructed from the perspective of noncooperative game. An iterative algorithm is then proposed to obtain the game cross efficiencies for the overall systems and subsystems. We use the proposed game model to evaluate the performance of top 30 US commercial banks. The results show that the game model can lead to a unique reasonable cross efficiency for each DMU.
\end{abstract}

\section{Introduction}

Data envelopment analysis (DEA) is a nonparametric approach for identifying the best practice of decision-making units (DMUs). Since it was firstly introduced by [1], there have been numerous studies in many areas, including profit and nonprofit organizations [2-6].

Conventional DEA models do not consider the internal structures and treat the whole production system as a black box. However, in many cases, DMUs may consist of twostage structures with intermediate measures, where all the outputs from the first stage are the only inputs to the second stage [7]. Great efforts have been devoted to evaluate the efficiencies of two-stage systems and their subsystems. Cook et al. [8] classified the existing models into four categories: standard DEA approach [9], efficiency decomposition approach [10, 11], network DEA approach [12], and gametheoretic approach $[13,14]$. Among them, the game-theoretic approach is quite meaningful and practical for two-stage systems, which can help decision makers to identify the inefficient individual subprocess and adjustment directions for each of the DMUs. The centralized model proposed by [13] is constructed from the perspective of cooperative game theory, where the two subsystems are viewed as one unity to maximize the efficiency of the whole system. This would be the case when the manufacturer and retailer jointly determine prices to achieve the maximum total profit. Under this framework, the efficiency of the whole system can be decomposed into the product of the efficiencies of the two subprocesses. When the optimal weights are not unique, there may exist some flexibility in decomposing the overall efficiency between the two subsystems. Liang et al. [13] and Kao [15] both suggested obtaining the maximal achievable efficiency of one stage firstly and computing the efficiency of the other stage subsequently. Zhou et al. [16] applied the Nash bargaining game theory to determine a fair and alternative decomposition when aforementioned flexibility exists. Du et al. [14] developed a Nash bargaining model under cooperative game assumption to evaluate the performance of DMUs with two-stage structures.

It is well known that one shortcoming of DEA is its extremely high flexibility in choosing optimal weights on input and output factors. A DMU can obtain a full efficiency by choosing very high weights on some factors and 
extremely low weights on other factors. This drawback may lead to unreasonable rankings of DMUs. This shortcoming also exists in the aforementioned game-theoretic models for DMUs with two-stage structures. Up to now, no literatures address this issue.

Cross efficiency evaluation, on the other hand, uses a peer-evaluation mode which is different from the selfevaluation mode of conventional DEA models. Under cross efficiency evaluation, mavericks have a lower chance of attaining high appraisal. The use of cross efficiency evaluation has been prevalent in many DEA application areas due to this merit $[3,4,17-19]$. Although cross efficiency has proven to be powerful in ranking DMUs, there still exist some drawbacks. The most important one is the nonuniqueness of virtual weights and cross efficiencies. Several approaches have been developed to alleviate this problem [20-25].

In order to solve the problems exist in the aforementioned game-theoretic centralized model for two-stage systems, that is, DMUs can choose very high weights on some factors and extremely low weights on other factors in order to obtain full efficiencies, we use peer evaluation instead of selfappraisal for the centralized model and define cross efficiency indicators for the whole system and two stages. We use peer-evaluation instead of self-appraisal for the centralized model and define cross efficiency indicators for the whole system and two stages. In order to solve the nonuniqueness of these indicators, a game model is constructed where DMUs with two-stage structures are regarded as players in a noncooperative game and the efficiency scores are viewed as payoffs. Each DMU chooses to take a game stance to the extent that it will attempt to maximize its payoff, which will lead to a unique cross efficiency.

The remainder of this paper is organized as follows. In the next section, the centralized model for two-stage systems is reviewed briefly. The definition of cross efficiency of the overall system and two subsystems is proposed. And then the DEA game cross efficiency model is developed together with the algorithm. In Section 3, the proposed model and algorithm are used to evaluate the performance of top 30 US commercial banks. Conclusions are provided in the last section.

\section{Game Cross Efficiency for Systems with Two-Stage Structures}

2.1. The Centralized Model Based on the Cooperative Game Concept. Consider a two-stage system shown in Figure 1. Suppose there are $n$ DMUs. For the $j$ th DMU, $X_{j}=$ $\left(X_{1 j}, \ldots, X_{m j}\right)^{T}$ represents the input vector to the first stage, and $Z_{j}=\left(Z_{1 j}, \ldots, Z_{t j}\right)^{T}$ represents the output vector from the first stage and also the only input vector to the second stage and is referred to as the intermediate vector. The output vector from the second stage is denoted by $Y_{j}=$ $\left(Y_{1 j}, \ldots, Y_{s j}\right)^{T}$.

If we ignore intermediate measures and treat the whole system as a black box, by using the constant return to scale

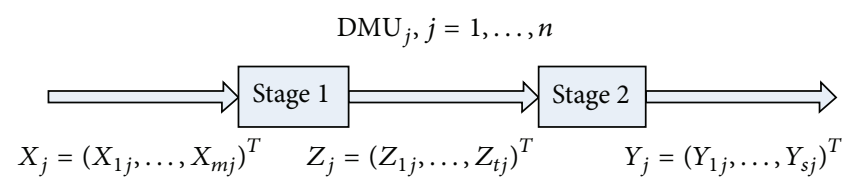

Figure 1: A system with two-stage structure.

(CRS) assumption, the efficiency score of the whole system for the $j_{o}$ th DMU, called $\mathrm{DMU}_{o}$ in short, can be calculated by the following CCR model:

$$
\begin{aligned}
& e_{o}=\max \frac{\sum_{r=1}^{s} u_{r o} Y_{r o}}{\sum_{i=1}^{m} v_{i o} X_{i o}} \\
& \text { s.t. } \begin{cases}\frac{\sum_{r=1}^{s} u_{r o} Y_{r j}}{\sum_{i=1}^{m} v_{i o} X_{i j}} \leq 1, & j=1, \ldots, n \\
v_{i o} \geq 0, & i=1, \ldots, m ; \\
u_{r o} \geq 0, & r=1, \ldots, s,\end{cases}
\end{aligned}
$$

where $v_{i}$ is the weight for the $i$ th input, $u_{r}$ is the weight for the $r$ th output, $i=1, \ldots, m$, and $r=1, \ldots, \mathrm{s}$.

Note that the above model only considers the initial inputs and final outputs and ignores intermediate measures; it may not properly characterize the performance of twostage systems, as discussed in [8]. For example, suppose that a DMU consists of a manufacturer and a retailer. In a cooperative environment, the manufacturer and retailer may wish to work together in determining price, order quantity, and other factors to achieve maximum savings and/or profit for the manufacturer-retailer supply chain. From this cooperative game concept, [13] proposed the following centralized model for evaluating the efficiencies of the whole system and subsystems simultaneously:

$$
\begin{aligned}
e_{o}=\max & \frac{\sum_{r=1}^{s} u_{r o} y_{r o}}{\sum_{i=1}^{m} v_{i o} x_{i o}} \\
\operatorname{s.t.} & \begin{cases}\frac{\sum_{d=1}^{D} w_{d o} z_{d j}}{\sum_{i=1}^{m} v_{i o} x_{i j}} \leq 1, & j=1, \ldots, n \\
\frac{\sum_{r=1}^{s} u_{r o} y_{r j}}{\sum_{d=1}^{D} w_{d o} z_{d j}} \leq 1, & j=1, \ldots, n \\
v_{i o} \geq 0, & i=1, \ldots, m ; \\
w_{d o} \geq 0, & d=1, \ldots, D ; \\
u_{r o} \geq 0, & r=1, \ldots, s .\end{cases}
\end{aligned}
$$


By using the "Charnes-Cooper" transformations in fractional programming [26], the above model can be converted to the following linear programming:

$$
\begin{aligned}
& e_{o}=\max \sum_{r=1}^{s} u_{r o} y_{r o} \\
& \text { s.t. } \begin{cases}\sum_{i=1}^{m} v_{i o} x_{i o}=1 \\
\sum_{d=1}^{D} w_{d o} z_{d j} \leq \sum_{i=1}^{m} v_{i o} x_{i j}, & j=1, \ldots, n \\
\sum_{r=1}^{s} u_{r o} y_{r j} \leq \sum_{d=1}^{D} w_{d o} z_{d j}, & j=1, \ldots, n \\
v_{i o} \geq 0, & i=1, \ldots, m ; \\
w_{d o} \geq 0, & d=1, \ldots, D ; \\
u_{r o} \geq 0, & r=1, \ldots, s .\end{cases}
\end{aligned}
$$

2.2. Definitions of Cross Efficiencies for Systems with Two-Stage Structures. Suppose that the optimal solution of Model (3) is $V_{o}^{*}=\left(v_{1 o}^{*}, \ldots, v_{m o}^{*}\right), W_{o}^{*}=\left(w_{1 o}^{*}, \ldots, w_{D o}^{*}\right)$, and $U_{o}^{*}=$ $\left(u_{10}^{*}, \ldots, u_{s o}^{*}\right)$ and the efficiencies of the overall system and two subsystems can be expressed as

$$
\begin{gathered}
e_{o}=\frac{\sum_{r=1}^{s} u_{r o}^{*} y_{r o}}{\sum_{i=1}^{m} v_{i o}^{*} x_{i o}}, \quad e_{o}^{1}=\frac{\sum_{d=1}^{D} w_{d o}^{*} z_{d o}}{\sum_{i=1}^{m} v_{i o}^{*} x_{i o}}, \\
e_{o}^{2}=\frac{\sum_{r=1}^{s} u_{r o}^{*} y_{r o}}{\sum_{d=1}^{D} w_{d o}^{*} z_{d o}} .
\end{gathered}
$$

For each $\mathrm{DMU}_{o}$ under evaluation, a set of optimal weights $V_{o}^{*}, W_{o}^{*}$, and $U_{o}^{*}$ are obtained. According to the cross efficiency definition in traditional DEA model, we give the following definition.

Definition 1. Let $V_{o}^{*}=\left(v_{1 o}^{*}, \ldots, v_{m o}^{*}\right), W_{o}^{*}=\left(w_{1 o}^{*}, \ldots, w_{D o}^{*}\right)$, and $U_{o}^{*}=\left(u_{1 o}^{*}, \ldots, u_{s o}^{*}\right)$ be an optimal solution to model (3). For each $\mathrm{DMU}_{j}$,

$$
\begin{gathered}
e_{o j}=\frac{\sum_{r=1}^{s} u_{r o}^{*} y_{r j}}{\sum_{i=1}^{m} v_{i o}^{*} x_{i j}}, \quad e_{o j}^{1}=\frac{\sum_{d=1}^{D} w_{d o}^{*} z_{d j}}{\sum_{i=1}^{m} v_{i o}^{*} x_{i j}}, \\
e_{o j}^{2}=\frac{\sum_{r=1}^{s} u_{r o}^{*} y_{r j}}{\sum_{d=1}^{D} w_{d o}^{*} z_{d j}}, \\
o, j=1, \ldots, n
\end{gathered}
$$

are called the o-cross efficiency of the overall system and two subsystems, respectively.
Definition 2. For each $\mathrm{DMU}_{j}$, the average of all $o$-cross efficiencies

$$
\begin{gathered}
E_{j}=\frac{1}{n} \sum_{o=1}^{n} e_{o j}, \quad E_{j}^{1}=\frac{1}{n} \sum_{o=1}^{n} e_{o j}^{1}, \\
E_{j}^{2}=\frac{1}{n} \sum_{o=1}^{n} e_{o j}^{2}, \\
j=1, \ldots, n
\end{gathered}
$$

are called the cross efficiency of the overall system and two subsystems, respectively.

It is evident that the overall o-cross efficiency can be decomposed into the product of the o-cross efficiencies of the two subsystems, and the overall cross efficiency can be decomposed into the average sum product of the o-cross efficiencies of the two subsystems. Note that Model (3) may have multiple optimal solutions; thus the cross efficiency may not be unique. Of course, we can make alternations to the aggressive and benevolent formulations developed by [21] for traditional DEA in order to alleviate this problem. One version of their approach is to find a weight bundle that maximizes or minimizes the average of the efficiencies of the other $n-1$ DMUs while keeping the efficiency of $\mathrm{DMU}_{o}$ unchanged. But this alleviation is limited, since multiple solutions may exist in the new aggressive (benevolent) model and cross efficiencies may still be not unique. In the following subsection, we will propose a model from the perspective of noncooperative game theory, which will lead to a unique cross efficiency.

2.3. Game Cross Efficiency Model for Systems with TwoStage Structures. As mentioned above, we can regard all DMUs as competitors in noncooperative game. In fact, each DMU may argue that its weight bundle should be chosen with a view to how that bundle impacts the implied performance of other DMUs. Conventional DEA achieves this by restricting the choice of bundles to those that keep the efficiencies of all DMUs no greater than unity. Cross efficiency carries out a new measure of efficiency in terms of not only the best multiplier bundle for the DMU itself, but also the best bundles for all the other DMUs.

Conventional cross efficiency models are constructed from the viewpoint of $\mathrm{DMU}_{o}$. Both of the aggressive and the benevolent formulations try to find a weight bundle that optimizes the average efficiencies of the other $n-1$ DMUs while remaining the efficiency of $\mathrm{DMU}_{o}$ at its ideal level. Here we deal with the problem from the viewpoint of competitors. For each competitor $\mathrm{DMU}_{j}$, a weight bundle is determined that maximizes the performance for itself, with the additional constraints that the resulting efficiency for $\mathrm{DMU}_{o}$ should be no less than its estimated best performance. Of course, we do not know the best performance of any DMU in advance. In the following subsection, we develop an iterative algorithm to obtain the best performance of all DMU simultaneously, in a cross efficiency sense. 
Assume that in a noncooperative game sense, one player $\mathrm{DMU}_{o}$ is given an efficiency score $\alpha_{o}$, and then another player $\mathrm{DMU}_{j}$ tries to maximize its own efficiency, subject to the condition that the efficiency of player $\mathrm{DMU}_{o}$ would not be decreased.

Definition 3. The game o-cross efficiency of overall system and two subsystems for $\mathrm{DMU}_{j}$ relative to $\mathrm{DMU}_{o}$ is defined as

$$
\begin{gathered}
\alpha_{o j}=\frac{\sum_{r=1}^{s} u_{r j}^{o *} y_{r j}}{\sum_{i=1}^{m} v_{i j}^{o *} x_{i j}}, \quad \alpha_{o j}^{1}=\frac{\sum_{d=1}^{D} w_{d j}^{o *} z_{d j}}{\sum_{i=1}^{m} v_{i j}^{o *} x_{i j}}, \\
\alpha_{o j}^{2}=\frac{\sum_{r=1}^{s} u_{r j}^{o *} y_{r j}}{\sum_{d=1}^{D} w_{d j}^{o *} z_{d j}}, \\
o=1, \ldots, n,
\end{gathered}
$$

where $V_{j}^{o *}=\left(v_{1 j}^{o *}, \ldots, v_{m o}^{o *}\right), W_{j}^{o *}=\left(w_{1 j}^{o *}, \ldots, w_{D j}^{o *}\right)$, and $U_{j}^{o *}=$ $\left(u_{1 j}^{o *}, \ldots, u_{s j}^{o *}\right)$ are the optimal solutions of the following game cross efficiency model:

$$
\begin{array}{ll}
\max & \sum_{r=1}^{s} u_{r j}^{o} y_{r j} \\
\operatorname{s.t.} & \begin{cases}\sum_{i=1}^{m} v_{i j}^{o} x_{i j}=1 & l=1, \ldots, n \\
\sum_{d=1}^{D} w_{d j}^{o} z_{d l} \leq \sum_{i=1}^{m} v_{i j}^{o} x_{i l}, & l=1, \ldots, n \\
\sum_{r=1}^{s} u_{r j}^{o} y_{r l} \leq \sum_{d=1}^{D} w_{d j}^{o} z_{d l}, & \\
\sum_{r=1}^{s} u_{r j}^{o} y_{r l} \leq \sum_{i=1}^{m} v_{i j}^{o} x_{i l}, & i=1, \ldots, n \\
\sum_{r=1}^{s} u_{r j}^{o} y_{r o} \leq \alpha_{o} \times \sum_{i=1}^{m} v_{i j}^{o} x_{i o}, & \\
v_{i j}^{o} \geq 0, & d=1, \ldots, D ; \\
w_{d j}^{o} \geq 0, & r=1, \ldots, s . \\
u_{r j}^{o} \geq 0, & \end{cases}
\end{array}
$$

In model (8), $\alpha_{o}$ is a parameter. In the following algorithm, we solve model (3) and obtain a set of initial cross efficiency scores $\alpha_{o}$ defined in (6). When the algorithm terminates, this $\alpha_{o}$ becomes the best game cross efficiency of $\mathrm{DMU}_{o}$. We refer to model (8) as the game model, in a noncooperative game and cross efficiency sense.
Definition 4. Let $V_{j}^{o *}=\left(v_{1 j}^{o *}, \ldots, v_{m o}^{o *}\right), W_{j}^{o *}=\left(w_{1 j}^{o *}, \ldots\right.$, $\left.w_{D j}^{o *}\right)$, and $U_{j}^{o *}=\left(u_{1 j}^{o *}, \ldots, u_{s j}^{o *}\right)$ be an optimal solution to model (8). For each $\mathrm{DMU}_{j}$,

$$
\begin{gathered}
\alpha_{j}=\frac{1}{n} \sum_{o=1}^{n} \sum_{r=1}^{s} u_{r j}^{o *} y_{r j}, \quad \alpha_{j}^{1}=\frac{1}{n} \sum_{o=1}^{n} \sum_{d=1}^{D} w_{d j}^{o *} z_{d j}, \\
\alpha_{j}^{2}=\frac{1}{n} \sum_{o=1}^{n} \frac{\sum_{r=1}^{s} u_{r j}^{o *} y_{r j}}{\sum_{d=1}^{D} w_{d j}^{o *} z_{d j}}
\end{gathered}
$$

are called the game cross efficiency of the overall system and two subsystems, respectively.

2.4. Iterative Algorithm for the Game Model. In this subsection, we propose an iterative algorithm for deriving the game cross efficiency scores. The basic idea of the algorithm is to start with the cross efficiency as defined in (6). For each $\mathrm{DMU}_{o}$, use the optimal objective function value of model (8) as the initial $\alpha_{o}$. This process is repeated for every $\mathrm{DMU}_{o}$, and the average of the optimal objective function values of model (8) becomes the new $\alpha_{o}$. When $\alpha_{o}$ falls in a specified small positive value $\varepsilon$ of one another, the algorithm terminates. The iterative algorithm is specified as follows.

Step 1. Solve model (3) and obtain a set of initial cross efficiency scores defined in (6). Let $t=1$ and $\alpha_{o, 1}=E_{o}$.

Step 2. Solve model (8) with $\alpha_{o}=\alpha_{o, 1}$ and obtain the optimal solution $V_{j}^{o *}=\left(v_{1 j}^{o *}, \ldots, v_{m o}^{o *}\right), W_{j}^{o *}=\left(w_{1 j}^{o *}, \ldots, w_{D j}^{o *}\right)$, and $U_{j}^{o *}=\left(u_{1 j}^{o *}, \ldots, u_{s j}^{o *}\right)$. Let

$$
\begin{gathered}
\alpha_{j, t+1}=\frac{1}{n} \sum_{o=1}^{n} \sum_{r=1}^{s} u_{r j}^{o *} y_{r j}, \quad \alpha_{j, t+1}^{1}=\frac{1}{n} \sum_{o=1}^{n} \sum_{d=1}^{D} w_{d j}^{o *} z_{d j}, \\
\alpha_{j, t+1}^{2}=\frac{1}{n} \sum_{o=1}^{n} \frac{\sum_{r=1}^{s} u_{r j}^{o *} y_{r j}}{\sum_{d=1}^{D} w_{d j}^{o *} z_{d j}} .
\end{gathered}
$$

Step 3. If $\left|\alpha_{j, t+1}-\alpha_{j, t}\right| \geq \varepsilon$ for some $j$, where $\varepsilon$ is a given small positive value, then let $\alpha_{o}=\alpha_{o, t+1}$ and go back to Step 2. If $\left|\alpha_{j, t+1}-\alpha_{j, t}\right|<\varepsilon$ for all $j$, then terminate. $\alpha_{j, t+1}, \alpha_{j, t+1}^{1}$, and $\alpha_{j, t+1}^{2}$ are, respectively, the game cross efficiency of the system and two subsystems for $\mathrm{DMU}_{j}, j=1, \ldots, n$.

Note that $\alpha_{o}$ initially takes the value defined in (6) in our algorithm. In fact, $\alpha_{o}$ can take other initial values, such as the aggressive or benevolent cross efficiencies defined in [21]. When the algorithm terminates, the same unique cross efficiency will be obtained.

\section{Application to Top 30 US Commercial Banks}

In this section, we will apply the game model to evaluate the performance of top 30 US commercial bank, which was used in Seiford and Zhu [9] first and then in Liang et al. [13, 22] and $\mathrm{Du}$ et al. [14]. The inputs of the first stage are number 
TABLE 1: Overall efficiencies from different models.

\begin{tabular}{|c|c|c|c|c|c|}
\hline DMU & Centralized & Arbitrary & Benevolent & Aggressive & Game \\
\hline 1 & 0.4487 (19) & $0.3386(25)$ & $0.3615(23)$ & $0.3305(25)$ & $0.4258(20)$ \\
\hline 2 & $0.3634(29)$ & $0.2735(29)$ & $0.2899(29)$ & $0.267(29)$ & $0.3428(29)$ \\
\hline 3 & $0.4216(23)$ & $0.3226(26)$ & $0.344(26)$ & $0.3161(26)$ & $0.4017(25)$ \\
\hline 4 & $0.4274(22)$ & $0.3389(24)$ & $0.3609(24)$ & $0.3334(24)$ & $0.4112(22)$ \\
\hline 5 & $0.6061(11)$ & $0.4016(16)$ & $0.4259(16)$ & $0.3957(16)$ & $0.529(12)$ \\
\hline 6 & $0.418(24)$ & 0.3544 (19) & $0.3733(19)$ & $0.3501(18)$ & $0.4069(23)$ \\
\hline 7 & $0.394(28)$ & $0.3113(28)$ & $0.3316(28)$ & $0.3058(28)$ & $0.3782(28)$ \\
\hline 8 & $0.4747(17)$ & $0.3553(18)$ & $0.3796(18)$ & $0.3474(19)$ & 0.4499 (17) \\
\hline 9 & $0.4486(20)$ & $0.3448(22)$ & $0.3638(22)$ & $0.3372(21)$ & $0.4232(21)$ \\
\hline 10 & $0.3393(30)$ & $0.2456(30)$ & $0.2639(30)$ & $0.2424(30)$ & $0.3178(30)$ \\
\hline 11 & $0.4158(26)$ & $0.3141(27)$ & $0.335(27)$ & $0.3072(27)$ & $0.3924(26)$ \\
\hline 12 & $0.4541(18)$ & $0.3449(21)$ & $0.3646(21)$ & $0.3368(22)$ & $0.4316(18)$ \\
\hline 13 & $0.4464(21)$ & $0.366(17)$ & $0.3872(17)$ & $0.3607(17)$ & 0.4287 (19) \\
\hline 14 & $0.4173(25)$ & $0.3512(20)$ & $0.3696(20)$ & $0.3462(20)$ & $0.406(24)$ \\
\hline 15 & $0.6421(9)$ & $0.4357(14)$ & $0.4498(14)$ & $0.4372(13)$ & $0.5053(14)$ \\
\hline 16 & $1(1)$ & $0.8478(2)$ & $0.8782(2)$ & $0.8377(2)$ & $0.9974(1)$ \\
\hline 17 & $0.7144(7)$ & $0.5787(5)$ & $0.6035(5)$ & $0.5726(5)$ & $0.6864(5)$ \\
\hline 18 & $0.7974(5)$ & $0.6709(4)$ & $0.6988(4)$ & $0.6662(4)$ & $0.7677(4)$ \\
\hline 19 & $0.5841(13)$ & $0.4648(12)$ & $0.479(12)$ & $0.4652(12)$ & $0.523(13)$ \\
\hline 20 & $0.5916(12)$ & $0.4983(7)$ & $0.5231(7)$ & $0.4918(8)$ & $0.5741(9)$ \\
\hline 21 & $0.5673(14)$ & $0.4712(11)$ & $0.4919(11)$ & $0.4653(11)$ & $0.5471(10)$ \\
\hline 22 & $0.5196(16)$ & $0.4382(13)$ & $0.4544(13)$ & $0.4365(14)$ & $0.4882(15)$ \\
\hline 23 & $0.3975(27)$ & $0.3398(23)$ & $0.3544(25)$ & $0.336(23)$ & $0.3882(27)$ \\
\hline 24 & $0.8257(3)$ & $0.7152(3)$ & $0.7504(3)$ & 0.7064 & 0.8085 \\
\hline 25 & $0.7403(6)$ & $0.4971(8)$ & $0.5146(8)$ & $0.4985(7)$ & $0.5823(8)$ \\
\hline 26 & $0.5306(15)$ & $0.431(15)$ & $0.4462(15)$ & $0.4305(15)$ & $0.4767(16)$ \\
\hline 27 & $0.6549(8)$ & $0.4791(10)$ & $0.497(9)$ & $0.4799(9)$ & $0.5419(11)$ \\
\hline 28 & $0.6198(10)$ & $0.5654(6)$ & $0.5898(6)$ & $0.5615(6)$ & $0.6173(7)$ \\
\hline 29 & $0.8093(4)$ & $0.4793(9)$ & $0.4935(10)$ & $0.4768(10)$ & $0.6267(6)$ \\
\hline 30 & $1(1)$ & $0.915(1)$ & $0.9473(1)$ & $0.9106(1)$ & $0.9971(2)$ \\
\hline
\end{tabular}

of employees, assets, and equity. The intermediate measures connecting the two subsystems are revenue and profit. The outputs from the second stage are market value and earning per share. See Seiford and Zhu [9] for detailed discussion on the above measures.

The efficiencies of the whole system from the centralized model and the cross efficiencies from the proposed game model $(\varepsilon=0.0005)$ are listed in Table 1 . Also, we apply the arbitrary, aggressive, and benevolent strategies developed by [21] for CCR model to the centralized model. The calculated cross efficiencies from different strategies are also reported in Table 1. All the corresponding ranks are listed in parentheses.

From Table 1, we can see that DMU16 and DMU30 perform efficiently for the centralized model. However, from the cross efficiency sense, none of DMUs are efficient. The largest difference between the second column and the last column lies in DMU15. It ranks 9 for the centralized model and 14 for the game model. If we look at the rankings from the game model and those under three strategies: arbitrary, aggressive, and benevolent, we will find that the difference is quite small. The reason lies on the fact that we use peer-appraisal instead of self-appraisal for the last four columns. Note that we construct our game model from the perspective of noncooperative game and each DMU is viewed as competitor. Thus, each competitor has an improved cross efficiency over that it received under the arbitrary, aggressive, and benevolent strategies, as shown in Table 1.

Table 2 reports the game cross efficiencies by using three different cross efficiencies under arbitrary, aggressive, and benevolent strategies as initial $\alpha_{o}$. From the overall efficiencies in Table 2, we can see that, although the iterations start from different $\alpha_{o}$, the calculated game cross efficiencies are almost the same. Similar phenomena occur in the game cross efficiencies of stage 1 and stage 2 . This indicates that our algorithm converges not only for overall game cross efficiency, but also for subsystems' cross efficiencies. Moreover, different from traditional cross efficiency definition, the proposed game cross efficiency is unique for the whole system and two subsystems.

Logically, the rank of the overall efficiency should lie between or in the neighborhood of the ranks of the efficiency scores of the two subsystems. For the 30 DMUs, 22 ranks lie 
TABle 2: Game cross efficiencies begin with different initial $\alpha_{o}$.

\begin{tabular}{|c|c|c|c|c|c|c|c|c|c|}
\hline \multirow{3}{*}{ DMU } & \multicolumn{9}{|c|}{ Game cross efficiency } \\
\hline & \multicolumn{3}{|c|}{ Arbitrary strategy } & \multicolumn{3}{|c|}{ Benevolent strategy } & \multicolumn{3}{|c|}{ Aggressive strategy } \\
\hline & Overall & Stage 1 & Stage 2 & Overall & Stage 1 & Stage 2 & Overall & Stage 1 & Stage 2 \\
\hline 1 & 0.4258 & 0.9852 & 0.4326 & 0.4257 & 0.9851 & 0.4325 & 0.4258 & 0.9852 & 0.4326 \\
\hline 2 & 0.3428 & 0.6670 & 0.5140 & 0.3427 & 0.6669 & 0.5139 & 0.3428 & 0.6670 & 0.5140 \\
\hline 3 & 0.4017 & 0.7812 & 0.5145 & 0.4016 & 0.7812 & 0.5145 & 0.4017 & 0.7813 & 0.5145 \\
\hline 4 & 0.4112 & 0.8496 & 0.4842 & 0.4111 & 0.8496 & 0.4842 & 0.4112 & 0.8496 & 0.4843 \\
\hline 5 & 0.5290 & 0.9916 & 0.5332 & 0.5286 & 0.9915 & 0.5329 & 0.5290 & 0.9916 & 0.5332 \\
\hline 6 & 0.4069 & 0.8035 & 0.5069 & 0.4068 & 0.8034 & 0.5069 & 0.4069 & 0.8035 & 0.5069 \\
\hline 7 & 0.3782 & 0.7674 & 0.4934 & 0.3781 & 0.7672 & 0.4933 & 0.3782 & 0.7674 & 0.4934 \\
\hline 8 & 0.4499 & 0.7339 & 0.6132 & 0.4498 & 0.7338 & 0.6131 & 0.4500 & 0.7339 & 0.6132 \\
\hline 9 & 0.4232 & 0.6877 & 0.6154 & 0.4231 & 0.6876 & 0.6153 & 0.4232 & 0.6877 & 0.6154 \\
\hline 10 & 0.3178 & 0.7212 & 0.4686 & 0.3177 & 0.7217 & 0.4680 & 0.3178 & 0.7212 & 0.4686 \\
\hline 11 & 0.3924 & 0.6455 & 0.6098 & 0.3922 & 0.6453 & 0.6097 & 0.3924 & 0.6455 & 0.6098 \\
\hline 12 & 0.4316 & 0.6770 & 0.6377 & 0.4314 & 0.6769 & 0.6376 & 0.4316 & 0.6770 & 0.6377 \\
\hline 13 & 0.4287 & 0.5685 & 0.7566 & 0.4286 & 0.5684 & 0.7566 & 0.4287 & 0.5685 & 0.7566 \\
\hline 14 & 0.4060 & 0.7047 & 0.5763 & 0.4060 & 0.7046 & 0.5762 & 0.4060 & 0.7047 & 0.5763 \\
\hline 15 & 0.5053 & 0.8166 & 0.6221 & 0.5051 & 0.8166 & 0.6218 & 0.5053 & 0.8166 & 0.6221 \\
\hline 16 & 0.9974 & 1.0000 & 0.9974 & 0.9973 & 1.0000 & 0.9973 & 0.9974 & 1.0000 & 0.9974 \\
\hline 17 & 0.6864 & 0.9845 & 0.6973 & 0.6862 & 0.9843 & 0.6972 & 0.6864 & 0.9845 & 0.6973 \\
\hline 18 & 0.7677 & 0.7677 & 1.0000 & 0.7676 & 0.7676 & 1.0000 & 0.7677 & 0.7677 & 1.0000 \\
\hline 19 & 0.5230 & 0.7347 & 0.7117 & 0.5228 & 0.7346 & 0.7116 & 0.5230 & 0.7347 & 0.7117 \\
\hline 20 & 0.5741 & 0.7472 & 0.7683 & 0.5740 & 0.7472 & 0.7682 & 0.5741 & 0.7472 & 0.7683 \\
\hline 21 & 0.5471 & 0.6413 & 0.8535 & 0.5470 & 0.6412 & 0.8535 & 0.5471 & 0.6413 & 0.8535 \\
\hline 22 & 0.4882 & 0.6595 & 0.7407 & 0.4881 & 0.6595 & 0.7405 & 0.4882 & 0.6595 & 0.7407 \\
\hline 23 & 0.3882 & 0.6249 & 0.6214 & 0.3881 & 0.6248 & 0.6213 & 0.3882 & 0.6249 & 0.6214 \\
\hline 24 & 0.8085 & 0.8681 & 0.9313 & 0.8084 & 0.8680 & 0.9312 & 0.8085 & 0.8681 & 0.9313 \\
\hline 25 & 0.5823 & 0.7241 & 0.8046 & 0.5821 & 0.7241 & 0.8043 & 0.5824 & 0.7241 & 0.8047 \\
\hline 26 & 0.4767 & 0.6256 & 0.7621 & 0.4766 & 0.6255 & 0.7620 & 0.4767 & 0.6256 & 0.7621 \\
\hline 27 & 0.5419 & 0.6389 & 0.8490 & 0.5417 & 0.6389 & 0.8488 & 0.5419 & 0.6389 & 0.8490 \\
\hline 28 & 0.6173 & 0.7715 & 0.8002 & 0.6173 & 0.7715 & 0.8002 & 0.6173 & 0.7715 & 0.8002 \\
\hline 29 & 0.6267 & 0.7774 & 0.8063 & 0.6262 & 0.7774 & 0.8058 & 0.6267 & 0.7774 & 0.8063 \\
\hline 30 & 0.9971 & 0.9971 & 1.0000 & 0.9970 & 0.9970 & 1.0000 & 0.9971 & 0.9971 & 1.0000 \\
\hline
\end{tabular}

between the ranks of the two subsystems and 6 ranks lie in the 2 rank neighborhoods, which conform to our intuition.

Figures 2, 3, and 4 show the iteration process of obtaining the best game cross efficiencies of the whole system and two subsystems for DMU10, respectively. Three cross efficiencies under arbitrary, aggressive, and benevolent strategies are used as initial $\alpha_{o}$ in our algorithm. As we can see, after 10 iterations, we find the best game cross efficiency for the whole system and two subsystems.

\section{Conclusion}

For systems with two-stage structure, the centralized model is the most popular approach used for measuring the performance, which is based on the concept of cooperative game for two subsystems. However, there exists too much flexibility in choosing optimal weights on input and output factors in the centralized model. A DMU can obtain a full

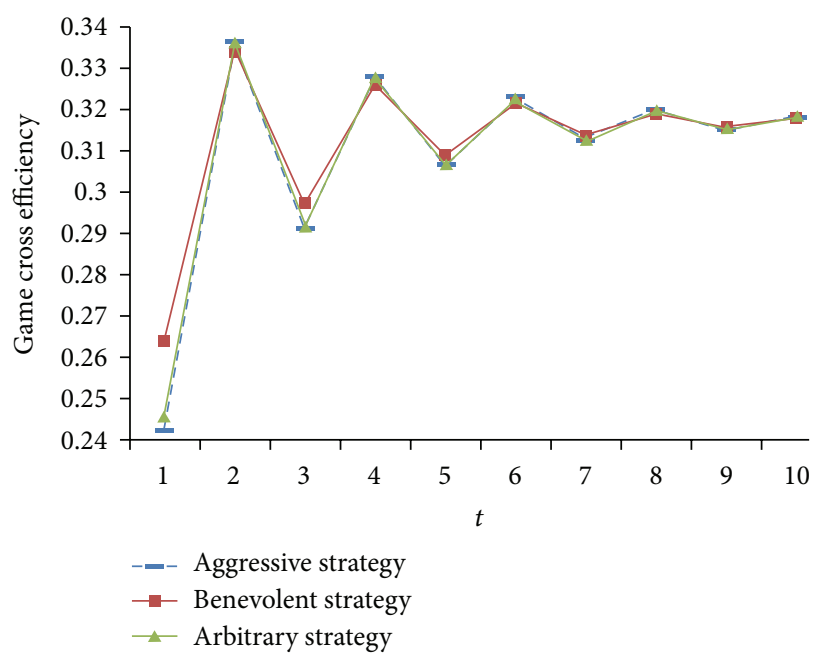

FIgURE 2: Iteration process for DMU10. 


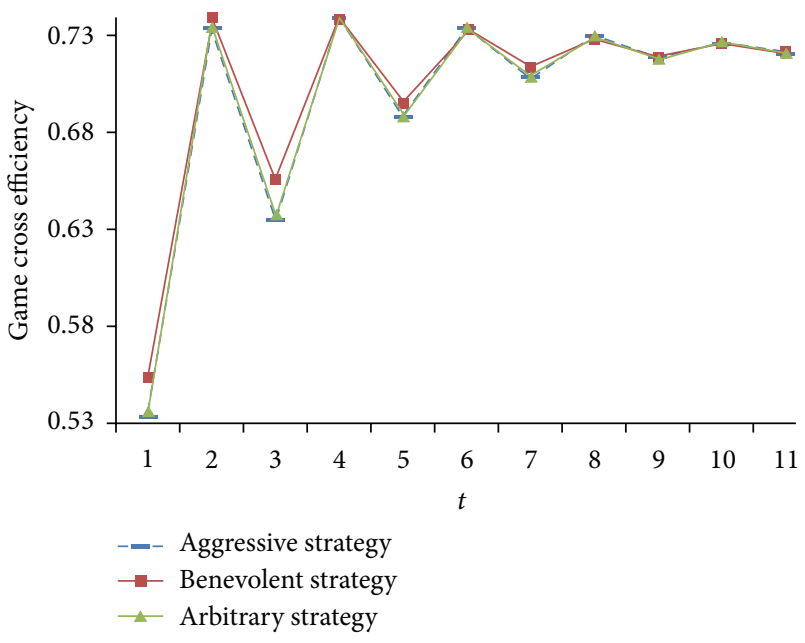

FIGURE 3: Iteration process for the first stage of DMU10.

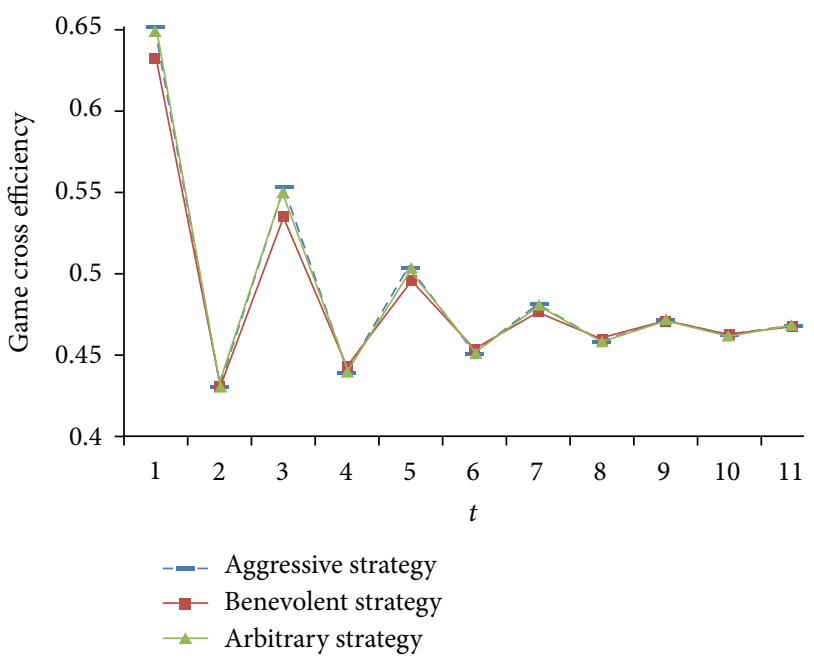

FIGURE 4: Iteration process for the second stage of DMU10.

efficiency by choosing very high weights on some factors and extremely low weights on other factors, which makes the ranking unacceptable. In order to solve the problem, we view DMUs as players in a game, and the efficiencies are viewed as payoffs. Each DMU chooses to take a game stance to the extent that it will attempt to maximize its payoff. From this viewpoint, we construct a game model. An iterative algorithm is developed to obtain the game cross efficiencies of the whole system and two subsystems. We use the proposed model and algorithm to evaluate the performance of top 30 US commercial banks. The results show that our model is more reasonable and the algorithm converges to a unique cross efficiency.

In practice, there are many systems comprising more than two subsystems, where all outputs of one subprocess must be the only inputs of the next subprocess. The formulation of our model shows that it can be extended to systems of multiple subsystems connected in series. Also, the algorithm can be modified accordingly in order to obtain the unique game cross efficiency.

\section{Conflict of Interests}

The authors declare that there is no conflict of interests regarding the publication of this paper.

\section{Acknowledgment}

This work is supported by Chinese National Natural Science Foundation (nos. 70901024, 71371067).

\section{References}

[1] A. Charnes, W. W. Cooper, and E. Rhodes, "Measuring the efficiency of decision making units," European Journal of Operational Research, vol. 2, no. 6, pp. 429-444, 1978.

[2] K. Nakabayashi and K. Tone, "Egoist's dilemma: a DEA game," Omega, vol. 34, no. 2, pp. 135-148, 2006.

[3] Z. Zhou, L. Zhao, S. Lui, and C. Ma, "A generalized fuzzy DEA/AR performance assessment model," Mathematical and Computer Modelling, vol. 55, no. 11-12, pp. 2117-2128, 2012.

[4] Z. Zhou, S. Lui, C. Ma, D. Liu, and W. Liu, "Fuzzy data envelopment analysis models with assurance regions: a note," Expert Systems with Applications, vol. 39, no. 2, pp. 2227-2231, 2012.

[5] Z. Zhou, W. Yang, C. Ma, and W. Liu, "A comment on, "A comment on 'A fuzzy DEA/AR approach to the selection of flexible manufacturing systems"' and, 'A fuzzy DEA/AR approach to the selection of flexible manufacturing systems,"' Computers \& Industrial Engineering, vol. 59, no. 4, pp. 1019-1021, 2010.

[6] S. Lozano, "DEA production games," European Journal of Operational Research, vol. 231, no. 2, pp. 405-413, 2013.

[7] Z. Zhou, M. Wang, H. Ding, C. Ma, and W. Liu, "Further study of production possibility set and performance evaluation model in supply chain DEA," Annals of Operations Research, vol. 206, no. 1, pp. 585-592, 2013.

[8] W. D. Cook, L. Liang, and J. Zhu, "Measuring performance of two-stage network structures by DEA: a review and future perspective," Omega, vol. 38, no. 6, pp. 423-430, 2010.

[9] L. M. Seiford and J. Zhu, "Profitability and marketability of the top 55 U.S. commercial banks," Management Science, vol. 45, no. 9, pp. 1270-1288, 1999.

[10] C. Kao and S.-N. Hwang, "Efficiency decomposition in twostage data envelopment analysis: an application to non-life insurance companies in Taiwan," European Journal of Operational Research, vol. 185, no. 1, pp. 418-429, 2008.

[11] Y. Chen, W. D. Cook, N. Li, and J. Zhu, "Additive efficiency decomposition in two-stage DEA," European Journal of Operational Research, vol. 196, no. 3, pp. 1170-1176, 2009.

[12] R. Färe and S. Grosskopf, "Productivity and intermediate products: a frontier approach," Economics Letters, vol. 50, no. 1, pp. 65-70, 1996.

[13] L. Liang, W. D. Cook, and J. Zhu, "DEA models for two-stage processes: game approach and efficiency decomposition," Naval Research Logistics, vol. 55, no. 7, pp. 643-653, 2008.

[14] J. Du, L. Liang, Y. Chen, W. D. Cook, and J. Zhu, "A bargaining game model for measuring performance of two-stage network 
structures," European Journal of Operational Research, vol. 210, no. 2, pp. 390-397, 2011.

[15] C. Kao, "Efficiency decomposition in network data envelopment analysis: a relational model," European Journal of Operational Research, vol. 192, no. 3, pp. 949-962, 2009.

[16] Z. Zhou, L. Sun, W. Yang, W. Liu, and C. Ma, "A bargaining game model for efficiency decomposition in the centralized model of two-stage systems," Computers \& Industrial Engineering, vol. 64, no. 1, pp. 103-108, 2013.

[17] G. N. Gregoriou, F. Rouah, S. Satchell, and F. Diz, "Simple and cross efficiency of CTAs using data envelopment analysis," European Journal of Finance, vol. 11, no. 5, pp. 393-409, 2005.

[18] J. Wu, J. Sun, L. Liang, and Y. Zha, "Determination of weights for ultimate cross efficiency using Shannon entropy," Expert Systems with Applications, vol. 38, no. 5, pp. 5162-5165, 2011.

[19] M. Falagario, F. Sciancalepore, N. Costantino, and R. Pietroforte, "Using a DEA-cross efficiency approach in public procurement tenders," European Journal of Operational Research, vol. 218, no. 2, pp. 523-529, 2012.

[20] T. R. Sexton, R. H. Silkman, and A. J. Hogan, "Data envelopment analysis: critique and extensions," New Directions for Program Evaluation, vol. 1986, no. 32, pp. 73-105, 1986.

[21] J. Doyle and R. Green, "Efficiency and cross-efficiency in DEA: derivations, meanings and uses," Journal of the Operational Research Society, vol. 45, no. 5, pp. 567-578, 1994.

[22] L. Liang, J. Wu, W. D. Cook, and J. Zhu, "The DEA game cross-efficiency model and its Nash equilibrium," Operations Research, vol. 56, no. 5, pp. 1278-1288, 2008.

[23] J. Wu, L. Liang, and Y.-C. Zha, "Determination of the weights of ultimate cross efficiency based on the solution of nucleolus," System Engineering Theory and Practice, vol. 28, no. 5, pp. 92-97, 2008.

[24] H. H. Örkcü and H. Bal, "Goal programming approaches for data envelopment analysis cross efficiency evaluation," Applied Mathematics and Computation, vol. 218, no. 2, pp.346-356, 2011.

[25] J. Wu, J. Sun, and L. Liang, "Cross efficiency evaluation method based on weight-balanced data envelopment analysis model," Computers \& Industrial Engineering, vol. 63, no. 2, pp. 513-519, 2012.

[26] A. Charnes and W. W. Cooper, "Programming with linear fractional functionals," Naval Research Logistics Quarterly, vol. 9, pp. 181-186, 1962. 


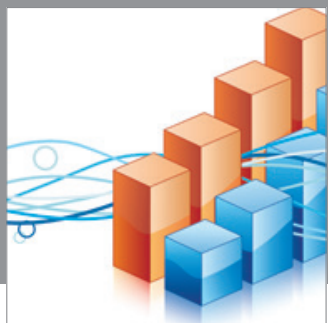

Advances in

Operations Research

mansans

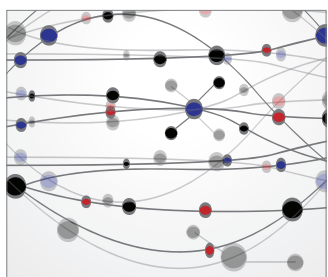

The Scientific World Journal
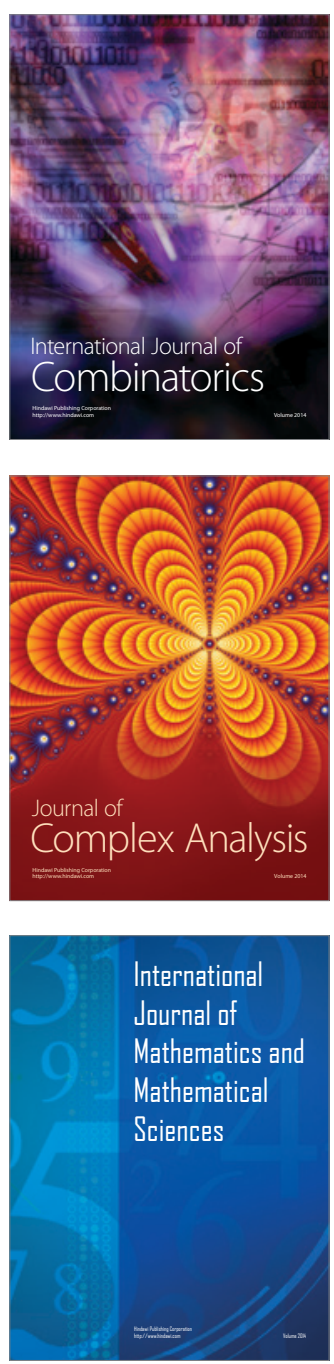
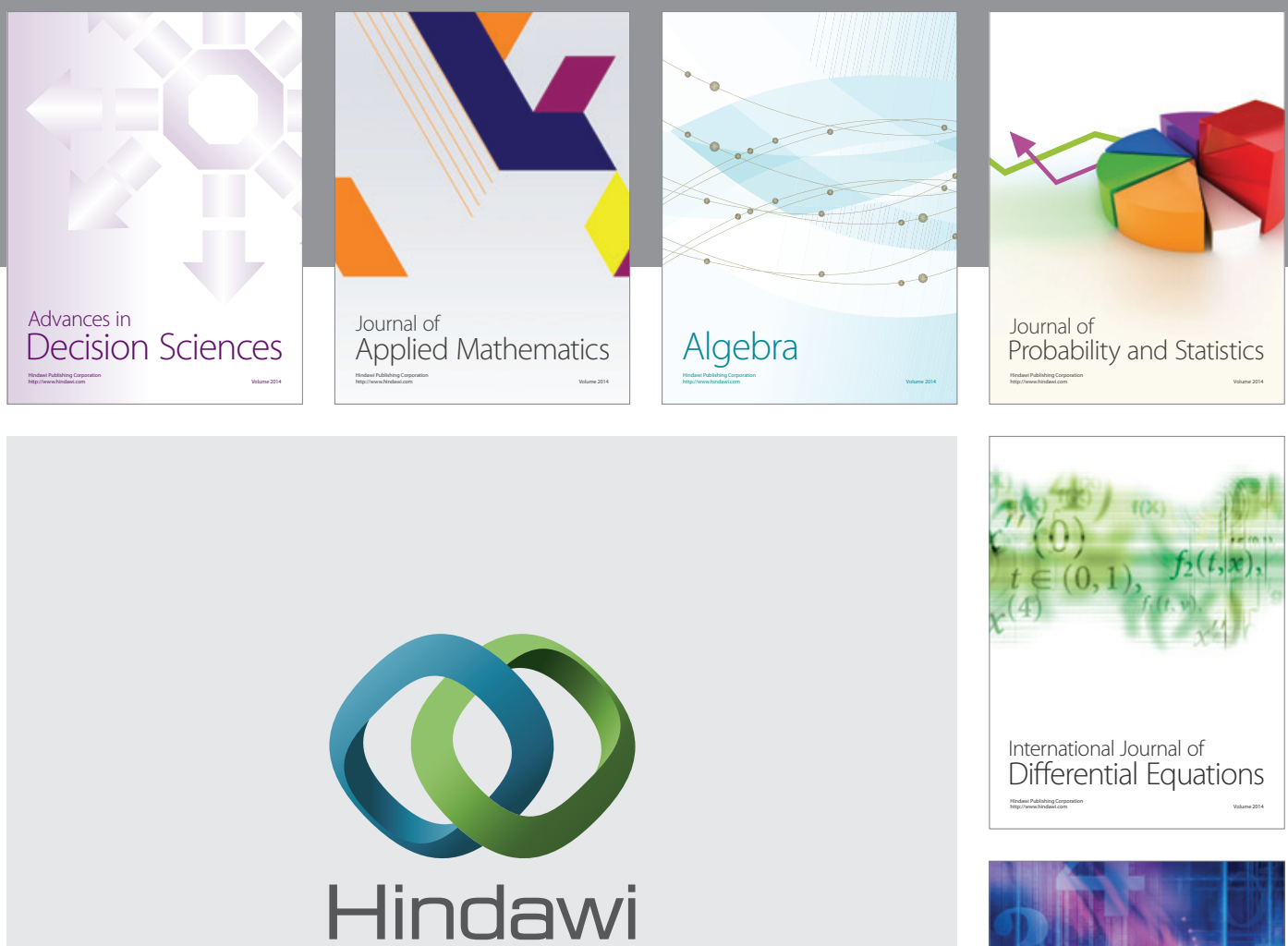

Submit your manuscripts at http://www.hindawi.com
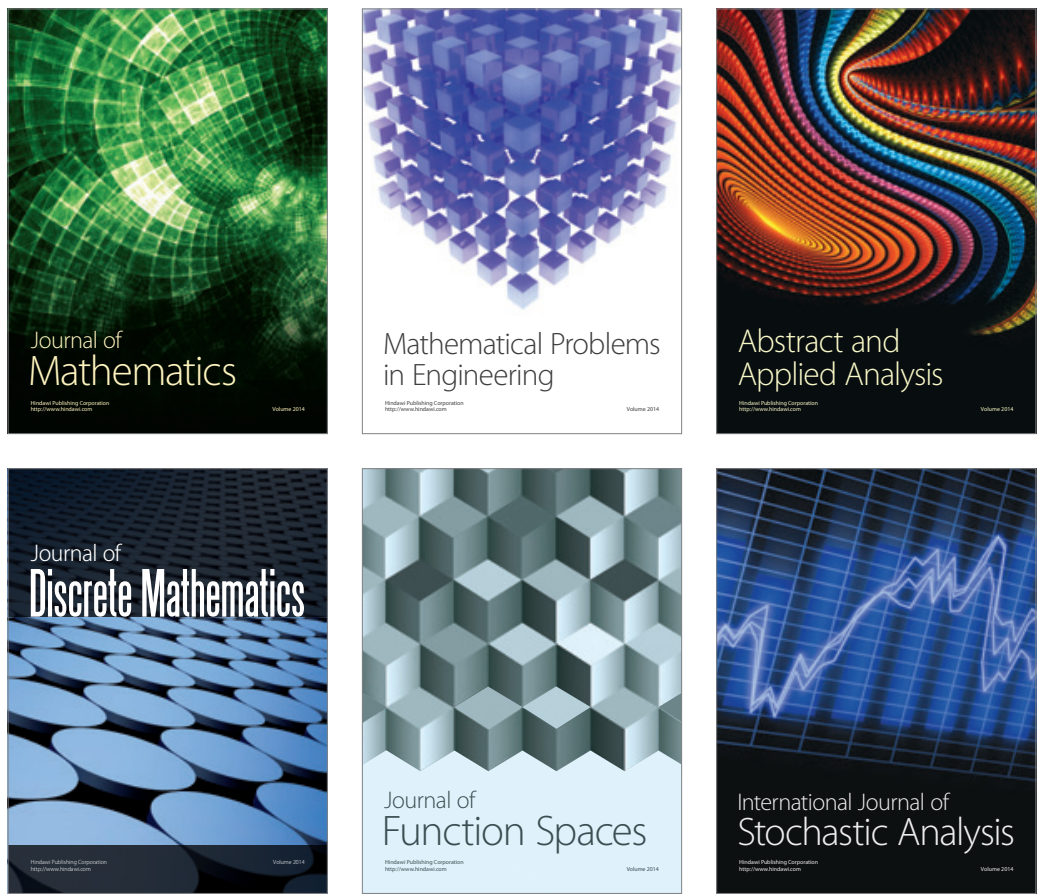

Journal of

Function Spaces

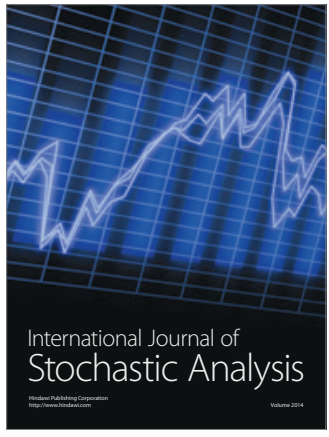

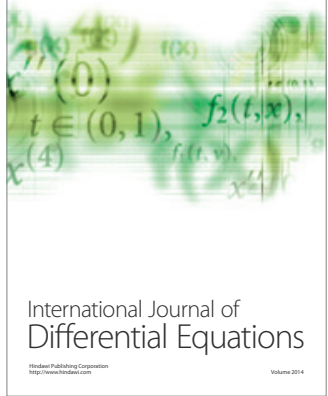
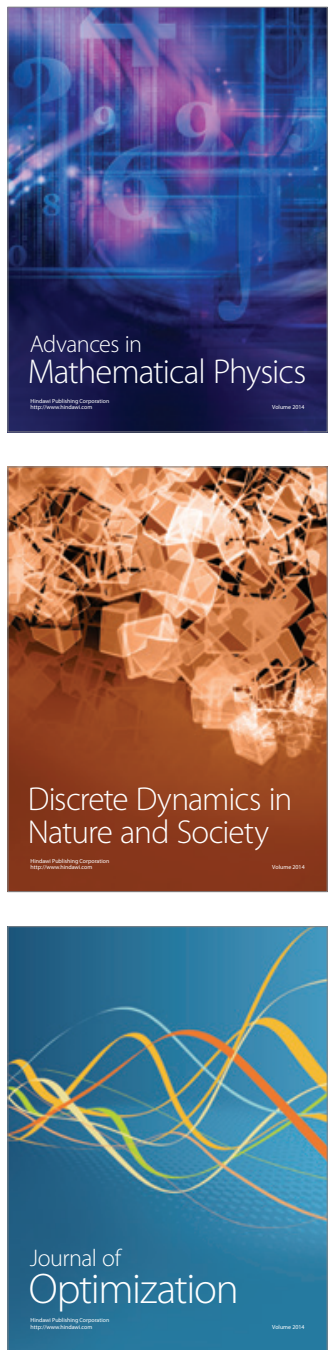